\title{
Hit the Thumb Jack! Using Electromyography to Augment the Piano Keyboard
}

\author{
Jakob Karolus ${ }^{1}$, Annika Kilian ${ }^{1 \dagger}$, Thomas Kosch ${ }^{1}$, Albrecht Schmidt ${ }^{1}$, Paweł W. Woźniak ${ }^{2 \ddagger}$ \\ ${ }^{1}$ LMU Munich, Munich, Germany \\ ${ }^{2}$ Utrecht University, Utrecht, the Netherlands \\ ${ }_{1}$ jakob.karolus, thomas.kosch, albrecht.schmidt\}@ifi.lmu.de, \\ †annika.kilian@campus.lmu.de, ’p.w.wozniak@uu.nl
}

\begin{abstract}
Improvising on the piano keyboard requires extensive skill development, which may reduce the feeling of immersion and flow for amateur players. However, being able to add simple musical effects greatly boosts a player's ability to express their unique playing style. To simplify this process, we designed an electromyography-based (EMG) system which integrates seamlessly into normal play by allowing musicians to modulate sound pitch using their thumb. We conducted an exploratory user study where users played a predefined melody and improvised using our system and a standard pitch wheel. Interview responses and survey answers showed that the EMG-based system supported the players' musical flow. Additionally, interviews indicated the system's capabilities to foster player creativity, and that players enjoyed experimenting with the effect. Our work illustrates how EMG can support seamless integration into existing systems to extend the range of interactions provided by a given interface.
\end{abstract}

\section{Author Keywords}

Electromyography; creative support tool; expressive piano play; seamless integration; motor tasks.

\section{CCS Concepts}

-Human-centered computing $\rightarrow$ Interaction techniques;

\section{INTRODUCTION}

When interacting with artifacts, we are often limited by the given range of possible interactions provided by the respective interface. Well-designed interfaces clearly communicate desired interactions and rely on expected movements. An example of such an interface is the piano keyboard, which is a well-established interface between the musician and the instrument. However, mastering the piano keyboard requires extensive skill development. Beginner and intermediate players are often limited to basic melodies and there is little room for improvisation. Accessing this aspect of musical play can

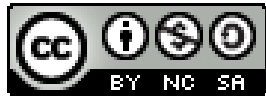

This work is licensed under a Creative Commons Attribution-NonCommercialShareAlike International 4.0 License.

DIS '20, July 6-10, 2020, Eindhoven, Netherlands. (C) 2020 Copyright is held by the author/owner(s).

ACM ISBN 978-1-4503-6974-9/20/07.

http://dx.doi.org/10.1145/3357236.3395500

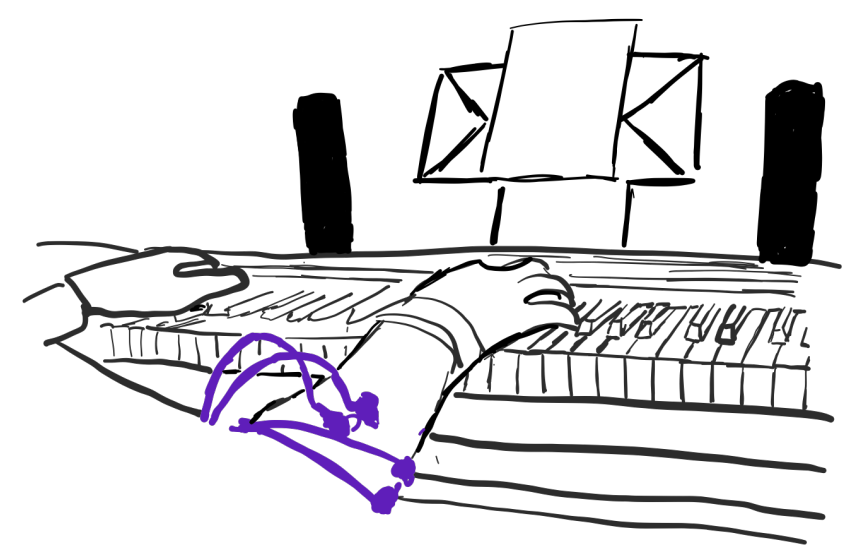

Figure 1. Sketch of a person playing the piano using our EMG-based system. Electrodes (purple) are connected to the forearm of the player.

be challenging as it is usually associated with increased costs, such as higher user workload.

In our work, we look at expressive capabilities of interaction modalities that subtly extend the range of interactions, hence allowing for seamless integration. For scenarios dominated by motor tasks such as playing the piano, electromyography (EMG) can serve as such a modality. EMG determines a person's muscular activity by means of measuring the electrical field through electrodes on the skin (surface EMG). Hence, the technique is able to either implicitly monitor activity or serve as an explicit input interface through muscle contraction. Especially for sensing-based interaction, it is vital that the range of sensed actions overlaps with the actions expected by the user [4]. To achieve this, we often make use of secondary modalities that work in tandem and supplement each other as it is the case with EMG and playing the piano.

The piano keyboard allows for a wide range of improvisation but suffers from one inherent problem: once the note is played, the musician cannot alter pitch nor dynamics. On an electronic keyboard a possible solution includes modulation wheels or auxiliary input switches. This, however, occupies at least one hand of the player. While research has already looked into adding pitch control to the piano keyboard [28], user augmentation via electromyography is yet to be evaluated. Measuring a person's muscle activity is not bound to a specific 
task, neither does it require prior preparation of the artifact, which often proves problematic for valuable instruments.

To verify the applicability of EMG in this scenario, we created a prototype that allows the pianist to modulate pitch during play by measuring their muscle activity. We implemented this experience by allowing the user to alter the pitch via a vibrato effect using a thumb wiggle gesture. To evaluate our approach, we conducted an experiment where users completed two tasks: playing a predefined tune and improvising using our EMGbased system and a standard pitch wheel. Our results confirm the technical feasibility of our concept and show that the EMGbased system supports the player during the improvisation stage by facilitating flow through seamless integration of the trigger gesture into the play for most participants. Electrodes were not perceivable and having access to a vibrato-effect provided players with an additional creative element when improvising.

We contribute the finding that Electromyography is a nonobtrusive modality that can be used effectively to facilitate improvisation on the piano keyboard, while not negatively influencing the musician's immersion and flow. Our results illustrate that these findings are not only limited to the piano keyboard, but allow EMG to be a secondary modality in most motor tasks, enabling additional input when manipulating tangible artifacts without altering the primary interface.

\section{RELATED WORK}

Research on the relationship between neural activity and muscle contraction dates back to the early 20th century, when Adrian and Bronk [1] utilized needle electrodes to measure muscle activation. In more recent research, EMG has predominantly been used in clinical applications such as prosthetic control [22,34] and detecting muscle diseases [2]. Due to the availability of affordable recording equipment, $\mathrm{HCI}$ has taken an interest in EMG as an input modality in the last decade, mainly focusing on auxiliary input methods which rely on specific hand and limb gestures to trigger muscle activity. In this section, we briefly introduce the physical background for EMG recording necessary throughout this paper and highlight relevant research projects conducted in recent years.

\section{EMG and $\mathrm{HCl}$}

The contraction of individual muscles allows us to direct our extremities and move our bodies. This contraction along the muscle fibers generates an electric field which can be measured on the skin via surface electromyography (sEMG). While this technique is more suitable for HCI purposes than using needle electrodes, penetrating several layers of tissue inevitably leads to a degradation of the signal $[27,30]$. Fortunately, most $\mathrm{HCI}$ applications do not require pinpointing individual muscle fiber activation, but rather rely on the ability to distinguish between different signal signatures. In 1988, Hefftner et al. $[21,20]$ explored autoregressive modelling to recognize EMG signatures. In their work, they mention common shortcomings and issues when working with EMG that still hold true today such as subject dependency and the need for training the muscle movements required for activation. In our work, we address the latter by focusing on electromyography as an auxiliary input modality that seamlessly integrates into the muscle movements of the primary task.

Implicit approaches include detecting emotions by recording facial muscles as showcased by Vrana [40]. Additionally, there has been a lot of research work towards utilizing EMG as an explicit input modality. Costanza et al. $[9,10]$ exploited an important property of isometric muscle activation, that is continuous activation but with no visible movement. Due to the discreet nature of the interaction, participants were able to navigate an audio menu using muscle contraction of the biceps. Observers were not able to detect any movements even when the participants were wearing short sleeves. This highlights the potential of EMG as a side-channel input modality.

\section{Advances in EMGxHCl Techniques}

EMG signals are measured as the potential between a ground electrode and a sensing electrode. Amplitude values range from $50 \mu V$ to $5 m V$ [6] creating the requirement for sensitive equipment. However, this makes EMG inherently noisy as the electrodes pick up electrical activity from other sources, e.g. secondary muscle movement, skin movement and environmental sources such as power line noise. Furthermore, when using a limited number of electrodes, the produced EMG signal can be quite ambiguous due to multiple muscle fibers contributing to the signal. [27, 30]

To reliably detect a set of gestures, numerous EMG sensing channels need to be deployed. In their work, Saponas et al. [35] employed a circular array of 10 electrodes (yielding 8 channels) around the forearm to distinguish gestures in four different sets with up to $95 \%$ accuracy. Yet they also report a high variance in detection accuracy for the different gesture sets and across participants. They showcase the potential for muCIs (muscle-computer interfaces) with purpose-built hardware. In their follow-up work [36], they additionally evaluated the approach in a more ecologically valid environment, demonstrating gesture classification was feasible even when the hand was already occupied, e.g. while carrying a bag or a mug. Karolus et al. [25] confirmed this expressive property of EMG for motor related tasks by employing it in a guitar tutor scenario. EMG estimated chord accuracy during play, helping the guitar player to choose an appropriate tempo setting. For our work, we closely follow related work by applying a sensing grid of 10 electrodes. Our aim is not to detect a set of predefined gestures but to recognize a given augmentation trigger during normal play.

\section{Expressiveness through Bio-Physical Devices}

The idea of using Biosensors as input controllers for music applications has been present in literature for a longer time. In 1990, Knapp and Lusted [26] proposed Biomuse, a bioelectric controller which used electroencephalography (EEG), electrooculography (EOG) and electromyography (EMG) as input modalities. Besides EMG, other bio-physical devices have been researched in the context of HCI, most notably in the domain of self-expression, e.g. as musical performance [32]. Donnarumma extensively explored mechanomyography (MMG) as one modality [14], while his recent work, 
together with his co-authors Caramiaux and Tanaka, also included EMG as an input channel [15, 7], contrasting the two technologies: EMG provided better locality.

Particularly for the piano keyboard, the term prepared piano is relevant, where artists explore everyday items to alter the piano sound ${ }^{1}$. In research, various augmentation methods and modalities have been explored [42, 17, 39], but not evaluated in studies. McPherson et al. [28] researched and evaluated expressive pitch control via capacitive sensors that reacted to the users' fingers touch and orientation. This allowed for great control over the used effect, but required extensive modifications to the piano.

Commercial products, such as the Neova ${ }^{2}$, the Seaboard RISE or the Lightpad $M^{4}$ promise beyond new forms of creativity, but are often tailored to support expert players. In our work, we leverage EMG as a means to increase the expressive range for amateurs without adding additional controls.

\section{Summary}

In recent years, EMG has proven to be a suitable input modality for human-centered applications both in terms of technical feasibility and expressive power. For the latter, we consider expression range (range of all possible interactions) and subjectively perceived expressiveness (what the user thinks they are capable of expressing). In our work, we focus not only on increasing the expressive range of the piano keyboard through an EMG-based system, but more importantly evaluate the impact of this augmentation in terms of the ability to create "musical ideas" - perceived expressiveness —, while putting an emphasis on seamless integration.

\section{RESEARCH QUESTIONS}

Our goal is to leverage electromyography as a secondary input channel, thus increasing the interaction range when playing the piano. To operationalize constraints, we formulate two research questions, each tailored towards a specific aspect, technical feasibility and user experience, of our work.

RQ1: Can a system using EMG accurately detect expressive finger motion without impacting the playing style, effectively increasing the interaction range?

This research question is two-fold: Firstly, it examines the technical feasibility of detecting finger motion via EMG. Here, we extensively draw on related work. Research has already demonstrated the suitability and potential of EMG [35, 25] for muscle-computer interfaces.

Secondly, we ask whether such a gesture can integrate seamlessly into the playing process. It has thus to be within the same domain of muscle movements, but have little overlap with the actual playing motion to prevent false positives. In other words: we wanted to design a gesture that the user would expect, but also one that can be sensed [4]. McPherson

\footnotetext{
${ }^{1}$ http://www . classical-music. com/article/

six-best-pieces-prepared-piano

2 https: //www . enhancia.co/

${ }^{3}$ https://roli. com/products/seaboard/rise-49

${ }^{4}$ https://roli . com/products/blocks/lightpad-m
}

et al. [28] investigated possible modulation gestures during piano play that had no to little overlap with existing techniques during play. The so-called space between the note [28] is of vital importance as it can be used to create new musical artifacts.

RQ2: What are design requirements for an EMG-based system which integrates seamlessly into existing motor tasks? What are the design factors that should be considered in a piano playing scenario?

We research the feasibility of "task language", i.e. finger motions that define the intended interaction ${ }^{5}$ - in contrast to the position of the fingers on the keys - to allow for playerspecific interaction. In other words, we focus on applying EMG to sense the aspects of an object that allow for providing contextual clues for extended interaction. This paradigm has been researched in other areas as well, such as PickRing [41] and work by Theiss et al. [37]. Analogously to the work by McPherson et al. [28], we extend on-the-move interaction through EMG, but do not limit the concept to specific objects, that need to be modified. Here, we envision the idea of seamless integration for existing tasks as detailed in the following design section.

Additionally, we look at the intrinsic motivation of people to play the piano and what drives them. How can we support this intrinsic motivation with our system to enable players to be more creative? Measuring creativity is challenging, since the term itself is ill-defined. To this end, Cherry and Latulipe [8] presented the Creativity Support Index (CSI); a validated score to measure creativity support, which we applied in our work. Here, we focus on the viability of an EMG-based modulation as a creativity support tool.

\section{DESIGN}

In the following section, we elaborate on our design decisions when building for seamless integration as well as contrast EMG with other sensing technologies to highlight its suitability for this work.

\section{Modalities for Seamless Integration}

Most prominent modalities include inertial measurement units (IMUs) and capacitive sensing [28] besides EMG. While all sensors can provide high accuracy, IMUs usually require the least amount of setup time. In contrast, capacitive sensing requires object modification and EMG requires to apply electrodes. Yet, IMUs struggle in sensing isometric muscle activation (applying pressure). Both capacitive sensing [28] and EMG [30] are able to do so, while the latter explicitly focuses on "user alteration" enabling interaction across devices and objects. We believe this to be an important design requirement to enable seamless integration.

\section{Design Aspects for Seamless Integration using EMG}

We identified three main design aspects based on our research questions that needed to be considered when employing EMG as a secondary input channel.

\footnotetext{
${ }^{5}$ E.g. playing an octave requires stretching of fingers.
} 


\section{Immersion and Flow}

Playing and creating music is closely associated with being in a state of flow and immersion [33, 12]. The effort required to achieve a state of flow is bound to play style and play proficiency. Hence, beginner or casual players struggle to find a compromise between self-expression through improvisation and the necessary mental demand. Especially for the piano, good knowledge of musical theory is required. Our EMGbased system aims to bridge this barrier by blending into the domain of playing movements — the task language allowing players to maintain a state of flow.

\section{Expressive Range and Control}

In our work, we benefit from McPherson et al.'s work [28], who already evaluated a suitable set of finger motions on the piano keyboard. We believe that allowing for a greater expressive range but retaining precise control is beneficial in our scenario. Thus, we employed only one modulation effect during our study as a fixed preset. In return, we allowed participants to define their own trigger gesture using their thumb, i.e. the inclination amplitude and direction of the motion as well as the applied pressure depending on individual preferences. This way our design allowed participants, despite being limited to thumb control, to define their own interaction with the piano keyboard owing to the flexibility offered by EMG.

\section{Comfort}

Comfort is closely related to immersion and flow as discomfort hinders these factors. It also connects to control, e.g. evoking discomfort when unreliable. Due to the nature of the system, electrodes need to be placed on the player's forearm to accurately record the electromyogram. Here, it is vital to evaluate how much the physical presence of the prototype and the used trigger gesture influences play.

\section{Prototype}

We implemented a thumb wiggle gesture as effect trigger: the user applies force on the piano key while simultaneously tilting his thumb back and forth. This motion then triggers a vibratoeffect (on or off) during play. Tilting direction and applied pressure are determined in a calibration phase and depend on individual preference and playing style. We allowed a variety of slightly different motions across our participants, as preliminary tests found no negative impact on detection accuracy. These tests also showed that using the thumb was most accurate and aligned well with occasions when one might want to add a vibrato effect ${ }^{6}$.

To record the electromyogram, we used BrainVision's LiveAmp EEG recorder ${ }^{7}$, allowing for wireless transmission to the recording PC. The system uses active surface electrodes to increase the signal-to-noise ratio including one ground (GND) and one reference (REF) electrode. Furthermore, active shielding reduced interference to electrical effects and artifacts due

\footnotetext{
${ }^{6}$ For example: ending a series of notes with a low key.

${ }^{7}$ https: //Ww . brainproducts . com/productdetails . php?id=63
}

to cable movement. A total of eight additional sensing electrodes were applied to the participants' right forearm and sampled at $500 \mathrm{~Hz}^{8}$.

Similar to related work (see $[35,25]$ ), electrodes were positioned in two rings around the arm and fixed with adhesive washers as shown in Figure 2. Each ring contained four data electrodes plus either GND or REF equally spaced around the forearm. This setup allowed to implicitly capture the relative electrode location and helped pinpoint local muscle activity. Prior to the start of the experiment, electrodes were injected with conductive gel and their impedance was checked.

The recorded data was transmitted to a recording computer and redirected as a data stream to the local network using $L a b$ Streaming Layer ( $L S L)^{9}$ ensuring sub-millisecond latency. Another computer was connected to an electronic piano keyboard via MIDI providing piano sound output via Ableton Live ${ }^{10}$ through external speakers in all conditions. Additionally, this computer connected to the available EMG stream for classification purposes. In our Electromyography condition, sound throughput was altered using MIDI signals to emulate a vibrato effect. The audio processing pipeline exhibits a latency of only a few milliseconds.

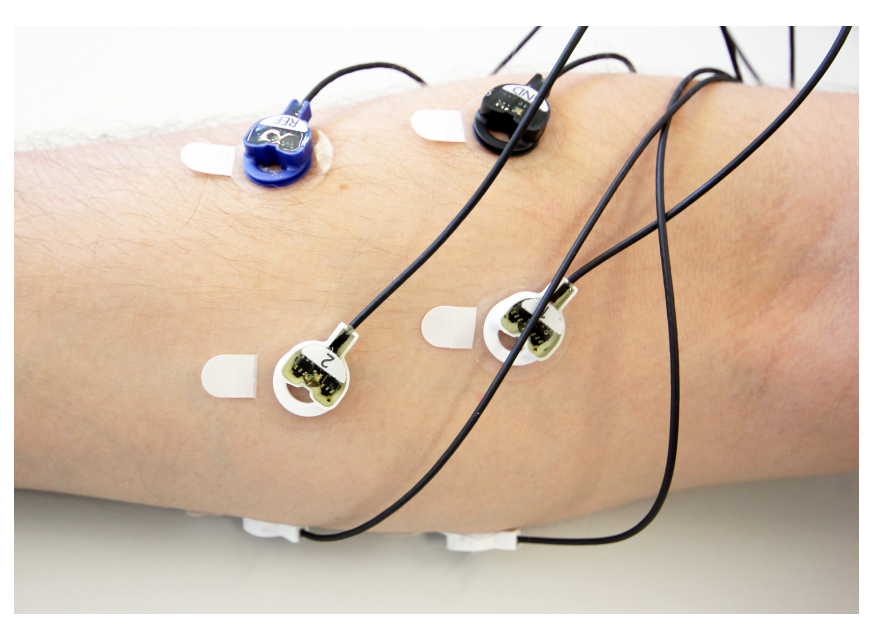

Figure 2. Example electrode configuration: two rings around the forearm each consisting of five electrodes (including either GND or REF).

\section{Data Preparation and Feature Generation}

For preprocessing and feature generation, we closely followed related work [30, 35, 25], applying the following filtering steps:

1. A fifth-order Butterworth bandpass filter between $2 \mathrm{~Hz}$ and $100 \mathrm{~Hz}$. This step is important to attenuate long-term drifts, the DC offset and high-frequency noise.

2. A fifth-order Butterworth bandstop filter to attenuate frequencies between $49 \mathrm{~Hz}$ and $51 \mathrm{~Hz}$ in order to remove power line interference.

\footnotetext{
${ }^{8}$ Depending on the movement one wants to recognize, different sampling rates are feasible [31]. For HCI purposes $500 \mathrm{~Hz}$ is adequate (see "Data Preparation" paragraph).

${ }^{9}$ https://github.com/sccn/labstreaminglayer

$10_{\text {https: / / www . ableton. com/en/ }}$
} 
A common analysis for time-series data is the segmentation into epochs [30]. Therefore, we divided the EMG signal into windows of fixed size for feature generation. Features based on the power of the signal have proven to be effective for classification and are computationally feasible [25, 35]. In this work, we rely on Root Mean Square (RMS) features. For a series $X=\left\{x_{1}, x_{2}, \ldots, x_{n}\right\}$, RMS is defined as

$$
x_{R M S}=\sqrt{\frac{1}{n}\left(x_{1}^{2}+x_{2}^{2}+\ldots+x_{n}^{2}\right)} .
$$

The RMS can be seen as a proxy for the intensity of muscle activity as the amplitude of the EMG signal increases when the muscular activity increases [30]. We computed the RMS for each channel as a separate feature. In addition, we computed the pair-wise ratios between all channels, yielding a total of 36 values for every window ${ }^{11}$.

Based on preliminary experiments, we set the window size to $150 \mathrm{~ms}^{12}$. Hop size was set to half the window size. Additionally, we submit all RMS features for the last and second-to-last window. Thus, for classifying the current state of the system, we look at a total of $300 \mathrm{~ms}$ worth of EMG samples. Note that classification is still performed every $75 \mathrm{~ms}$ (hop size). We found that this method ensured stable classification while also maintaining decent latency. No participant reported any perceived activation latency when asked about it in the interviews.

\section{Classification}

Classification was performed using a support vector machine using a radial basis function kernel ${ }^{13}$. Ground truth labels for classification were assigned during calibration ${ }^{14}$ (effect ON/OFF), resulting in a binary classification scheme. During the calibration phase, the experimenter generated ground truth by providing labels for the EMG data by indicating start and end of an effect period. In a real-world context, this could be provided by the player themselves during warm-up exercises. A five-fold cross validation served as a sanity check after calibration to indicate classification performance.

\section{EVALUATION}

We evaluated our prototype and the aforementioned design aspects in an experiment consisting of two different tasks. The first task Notesheet required the user to play a simple piece from notes and modulate at priorly defined positions. This task specifically aimed to assess the technical feasibility of the approach, while also introducing the participants to our system and its operating principles. The second task Improvisation then allowed the participants to fully explore the prototype and incorporate its features into their own playing style. They were free to improvise at will or play pieces from memory or from notes. In contrast to Notesheet, we explicitly evaluated the effectiveness of our prototype as a creative support tool in this task and addressed design aspects in a post-hoc interview.

\footnotetext{
${ }^{11} 8$ channels plus 28 pair-wise ratios.

${ }^{12}$ Corresponds to 75 samples.

${ }^{13} \mathrm{C}=1.0, \gamma=$ scale as evaluated a priori. Also see sckit-learn (https: //scikit-learn.org/stable/index.html)

${ }^{14}$ See "Procedure" section.
}

\section{Study Design}

To allow for comparison, we introduced an auditory baseline that offered the same expressive range albeit using a different modality. Hence, we employed a within-subject design with one independent variable Modality for each task. The two conditions differed in their way of triggering the modulation. The Pitch Wheel $(P W)$ condition required the participants to modulate pitch via the pitch wheel, manually creating a vibrato effect. The pitch wheel represents a standard control mechanism, available in most equipment used by amateur players. The second condition, Electromyography $(E M G)$, relied on the calibrated trigger gesture to activate an automatic vibrato effect. Conditions were counter-balanced within each task. Using two tasks of varying difficulty allowed us to evaluate the system in different scenarios.

\section{Measures}

Our data collection focused on three different aspects of our work: 1) we measured objective detection performance for our prototype, 2) we asked participants about creativity aspects as well as their inherent motivation and 3) we asked participants follow-up questions and interviewed them to capture aspects not covered by the previous questionnaires. Details about these measures and their analysis are provided in the following sections.

\section{NASA TLX}

The NASA TLX questionnaire [19] is a standardized test for perceived workload. It is divided into six subscales: mental demand, physical demand, temporal demand, performance, effort and frustration. We employed the NASA TLX in its raw form excluding the weighting process [18] as a manipulation check for our two tasks Notesheet and Improvisation to evaluate our application of two different levels of difficulty.

\section{Hedonic and Eudaimonic Motives for Activities (HEMA)}

To get a basic understanding of what drives people to play the piano, we relied on the "Hedonic and Eudaimonic Motives for Activities" survey [23]. The questionnaire has already been in the focus of user experience research [29]. This allowed us to see if our participants primarily pursued hedonic (momentary pleasure) or eudaimonic aspects (lasting meaning) giving insight into how they might integrate our prototype into their playing style.

\section{Creativity Support Index (CSI)}

The "Creativity Support Index" (CSI) survey [8] was developed to evaluate the ability of a tool to support a user engaged in creative work. The administration closely follows the NASA TLX and focuses on six dimensions of creativity support: exploration, expressiveness ${ }^{15}$, immersion, enjoyment, results being worth the effort, and collaboration. Resulting scores are weighted by these factors and give an indication of a tool's assistive capabilities in terms of creative work. The weight assigned to individual factors additionally highlights aspect of creativity support that are more important to users than others. We decided to administer the CSI survey for our second Task Improvisation to measure the creativity support for the two employed conditions Pitch Wheel and Electromyography, thus

\footnotetext{
${ }^{15}$ Covers expressive range and perceived expressiveness.
} 
Questions assessing perceived accuracy and intuitiveness

A1. Please rate your perceived accuracy of the system in recognizing your augmentation.

A2. How intuitive was the gesture movement?

A3. How intuitive was the sound modulation?

A4. How intuitive was the combination between the gesture movement and the sound modulation?

Table 1. Additional questions for both conditions $(P W, E M G)$ and both tasks (Notesheet, Improvisation); all visual analog scale (0 to 100).

Self-efficacy and tool support questions

B1. I am good at coming up with new ideas.

B2. I have a lot of good ideas.

B3. I have a good imagination.

C1. The tool supported me in coming up with new ideas.

C2. The tool supported my good ideas.

C3. The tool fostered using my imagination.

Table 2. Questions polling self-efficacy and tool support; all visual analog scale (0 to 100). Question B1-3 [3] were administered for Improvisation once. Questions C1-3 were administered for both conditions during Improvisation.

tasking participants to fill out the agreement statements after each condition, and eventually administering the paired-factor comparison [8]. We omitted the factor collaboration in our analysis as it is not within the scope of this work ${ }^{16}$.

\section{Custom Questionnaire}

We constructed a set of questions about the perceived accuracy and intuitiveness of the activation movements and the respective sound modulation as shown in Table 1. All answers were recorded on a visual analog scale ranging from 0 to 100. This questionnaire was administered for both conditions and both tasks. Exclusively for the Improvisation task, we administered three questions focusing on the subject's own creative self-efficacy as defined by Tierney \& Farmer [38]: one's ability to generate novel and useful outcomes. An operationalized version can be found in Beghetto's work [3] (see Table 2: Questions B1-B3). Each condition was then complemented by an adapted version that evaluates a tool's creative support as shown in Table 2 (Questions C1-C3).

\section{Interviews}

In a post-hoc interview, we asked the participants about their experience when using the two different conditions for sound modulation. Specifically, we asked them to contrast the feeling of integration for both conditions and how either had impacted their play style and perceived expressiveness when improvising. Each interview took approximately 10 minutes.

\section{Procedure}

We introduced our prospective participants to the experiment and handed them a detailed description explaining each task and the intended procedure. Participants were advised that the

\footnotetext{
${ }^{16}$ The CSI survey specifically allows for this configuration.
}

muscle activity of their right forearm would be recorded, requiring the placement of adhesive electrodes. After providing informed consent, the participants completed a demographics questionnaire which included gender, age and piano playing experience. The experimenter was present to answer any questions.

Before the start of the experiment, the experimenter placed the electrodes on the right forearm. As outlined previously, the first task Notesheet required participants to play a piece from a sheet of music. Here, participants could choose between simplified versions of either Ode to Joy or Conquest of Paradise. Both songs included marked notes throughout the piece where the player was required to use a vibrato effect. Long notes and notes ending a descending run were chosen for this purpose. As required by the $E M G$ condition, calibration for the trigger gestures was done directly while playing the chosen song ${ }^{17}$ twice in different registers, lasting no more than two minutes. This calibration was always executed first. Depending on condition order, the vibrato effect had to be applied using the pitch wheel or the EMG-based trigger gesture. Each condition was completed by filling out the NASA TLX and our custom questionnaire (Questions A1-A4).

Before starting the second task, we recorded the answers to the HEMA survey and Question B1-B3 from our questionnaire. If required, a recalibration was executed before beginning the task, at the discretion of the experimenter. Task Improvisation allowed the participant to experiment with both conditions during a free improvisation session. Additionally, we provided a library of musical pieces ${ }^{18}$ for use if needed. Participants were given approximately five minutes per condition, followed by filling out the agreement statement of the CSI survey and Questions C1-C3 from our custom questionnaire, and answering the paired-factor comparison of the CSI survey. A semistructured interview concluded the experiment. The whole experiment, including electrode placement, did not exceed one hour. Ethical approval for this study was obtained from the Ethics Committee at the University of Constance.

\section{Participants}

We recruited twelve ${ }^{19}$ participants (9m, 3f; age $\bar{x}=23.2 y$, $S D=3.0 y$ ) from the University of Munich via mailing lists. The data of nine were used for the complete analysis, while the data of three participants $(2 \mathrm{~m}, 1 \mathrm{f})$ were only used for the qualitative analysis due to detected outliers ${ }^{20}$. Although subjective play proficiency varied $(\bar{x}=32.0, S D=19.6 y)^{21}$, all participants were able to play a piece from the written notes and reported an average of $\bar{x}=11.2 \mathrm{~h} / \mathrm{month}(S D=11.2 \mathrm{~h} / \mathrm{month})$ spent playing the piano. After the study, each participant was reimbursed with 10 euros.

\footnotetext{
${ }^{17}$ Participants were given time to practice the given song if required.

${ }^{18} \mathrm{~A}$ personal collection available at the office.

${ }^{19}$ Since all participants are familiar with the instrument, we believe this to be a meaningful sample size for our evaluation [24].

${ }^{20}$ Social desirability bias and technical reasons.

${ }^{21}$ Visual analog scale from 0 to 100.
} 


\section{RESULTS}

We report on collected classification accuracy during the calibration phases, the outcomes of the presented surveys ${ }^{22}$ and their quantitative analysis, and present a qualitative analysis of the conducted interviews.

\section{Prediction Accuracy}

Using a ten-fold cross validation we achieved an average accuracy (over all participants) of $91 \%(S D=6 \%)^{23}$ for calibration. The lowest score was $81 \%$, the highest was $97 \%$. As mentioned in the procedure section, these measurements were used to assess the quality of the calibration during the experiment. Reporting exact classification accuracy during the Improvisation was not possible, since collecting ground truth was not feasible. Furthermore, reducing electrode count to one channel, only slightly impacts prediction performance, still achieving an average accuracy (over all participants and all channels) of $86 \%(S D=7 \%, \min =73 \%, \max =96 \%)$.

\section{Perceived Workload (NASA TLX)}

Improvisation exhibited a higher perceived workload as measured by the raw NASA TLX score at $\bar{x}=59.17(S D=18.46)$ compared to Notesheet at $\bar{x}=45.00(S D=21.86)$ as intended by the study design. A paired sample t-test showed a significant effect of Task: $t(8)=-3.10, p<.05, r=.73$. No deviation from normality was confirmed visually and via the Shapiro-Wilk test $(W=0.89, p>.05)$.

\section{HEMA survey}

As outlined previously, the results of the HEMA survey [23] helped us identify the main motivation for playing the piano. Items ${ }^{24}$ in the survey are rated from 1 (not at all) to 7 (very much). Most of our participants value hedonic ( $\bar{x}=5.33, S D=$ $1.0)$ over eudaimonic $(\bar{x}=4.38, S D=2.1)$ motives when playing the piano. It is noteworthy that they do appreciate a challenge as the question "Seeking to take it easy?" only recorded a mean of $\bar{x}=3.58(S D=1.56)$.

\section{CSI scores}

In order to determine the most useful creativity aspects, we analyzed factor weights and examined their individual scores as suggested by Cherry et al. [8]. High factor weights indicate that this aspect was especially important to participants. Weights range from 0 to 5 and are calculated based on the paired-factor comparison administered at the end of the experiment. Expressiveness was ranked highest with 3.44, followed by exploration (3.22). The other factors were of moderate importance for the participants. An overview can be seen in Table 3.

Figure 3 depicts the aggregated scores from the agreement statements ${ }^{25}$ of the CSI survey. The maximum factor score

\footnotetext{
${ }^{22}$ Survey data is available as supplementary material.

${ }^{23}$ Leave-one-participant-out validation: $86 \%(S D=7 \%), \min =75 \%$, $\max =96 \%$.

${ }^{24} \mathrm{~A}$ total of nine questions; five for hedonic motives and four for eudaimonic motives.

${ }^{25}$ Two agreement statements per factors, averaged over participants.
}

\begin{tabular}{lc}
\hline Factor & Weight \\
\hline Enjoyment & 2.33 \\
Exploration & 3.22 \\
Expressiveness & 3.44 \\
Immersion & 2.67 \\
ResultsWorthEffort & 2.56 \\
\hline
\end{tabular}

Table 3. Factors in the CSI survey and their allocated weight by participants.

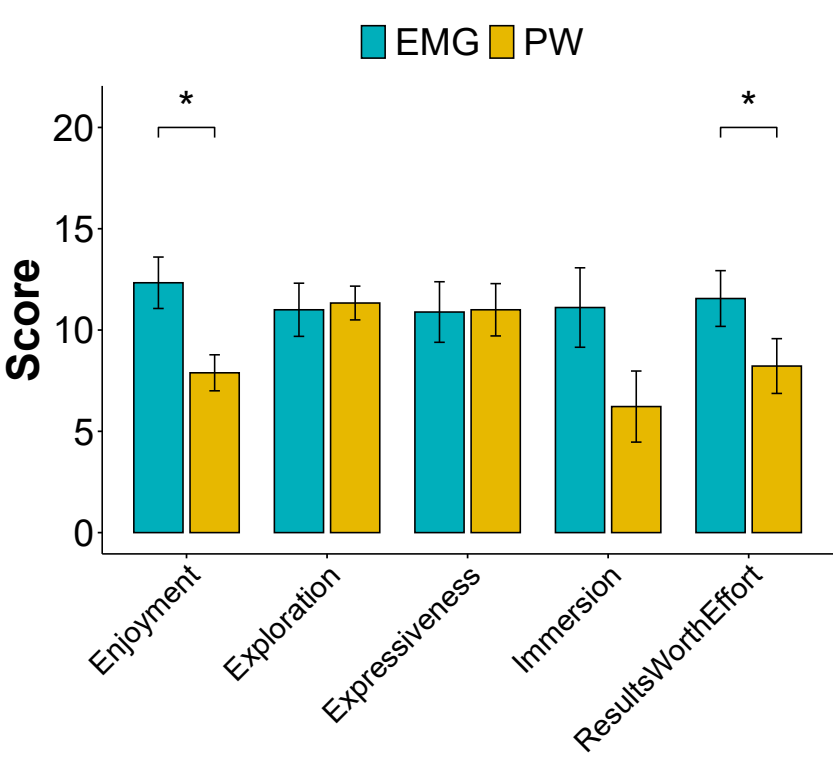

Figure 3. Mean scores for individual factors of the CSI survey grouped by condition $(E M G, P W)$. Error bars show standard error. Significant differences between conditions are marked with *.

is 20 . The two most important factors ${ }^{26}$ : exploration and expressiveness showed similar scores for both conditions. $E M G$ outperforms $P W$ for enjoyment, immersion and results worth effort. A Wilcoxon signed ranked test confirmed that using the EMG-based system led to significantly higher scores for enjoyment $(V=41, p<.05, r=.57)$ and results worth effort $(V=37, p<.05, r=.47)$.

\section{Custom questionnaire}

Results on gesture movement and sound modulation are illustrated in Figure 4 on a visual analog scale (0 to 100). Please refer to Table 1 for the respective question identifier.

A paired sample t-test showed a significant effect of Modality for Question 3 ("How intuitive was the sound modulation?") and Question 4 ("How intuitive was the combination between the gesture movement and the sound modulation?"): $t(8)=3.00, p<.05, r=.72$ and $t(8)=3.01, p<.05, r=.73$, respectively. No deviation from normality was confirmed visually and via the Shapiro-Wilk test $(W=0.91, p>.05$ and $W=0.85, p>.05$, respectively).

Our additional questions (B1-B3, C1-C3) which investigated self-efficacy and their adapted version are shown in Figure 5.

26 as indicated by their assigned weights 


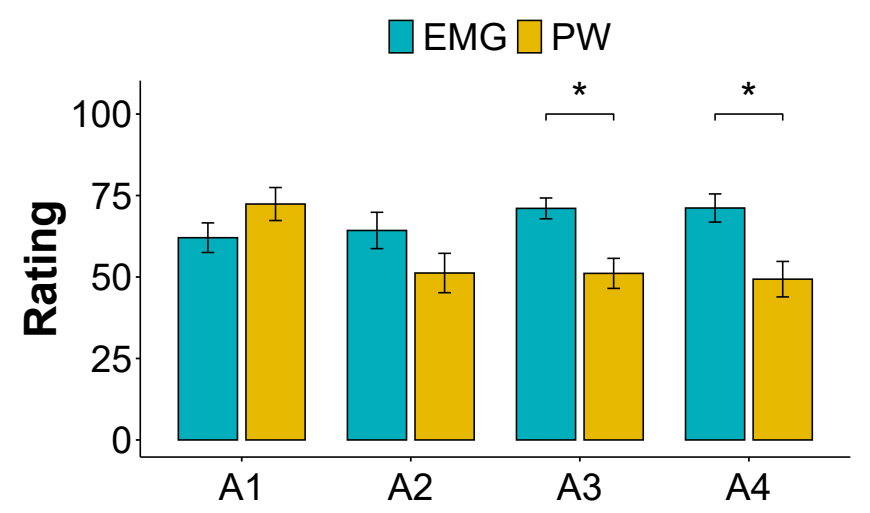

Figure 4. Mean scores for Question A1-A4; assessing perceived accuracy and intuitiveness of both conditions (see Table 1). Error bars show standard error. Significant differences between conditions are marked with *.
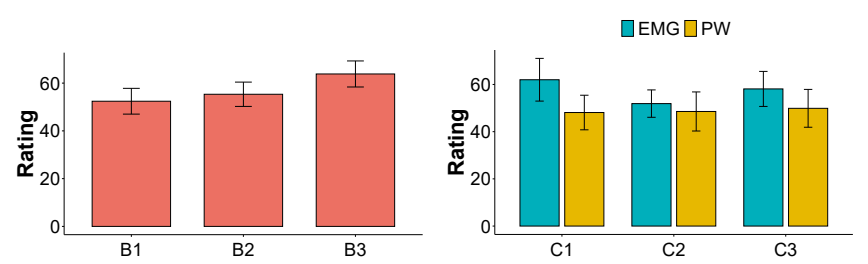

Figure 5. Mean scores for questions assessing self-efficacy of our participants (left side: B1-B3) and tool support (right side: C1-C3), see Table 2. Tool support is measured for each condition. Error bars show standard error.

For Questions C1 through C3, no significant differences between the conditions were found.

\section{Interviews}

All interviews were recorded (total duration $1: 41 h$ ) and transcribed verbatim. As the volume of the data was comparably low and we wanted to conduct a focused analysis, we opted for the pragmatic approach to thematic analysis as described by Blandford et al. [5]. Two researchers coded a representative $20 \%$ of the material. We then developed an initial coding tree based on iterative discussion. The rest of the interviews were then evenly split between the two coders. In a final discussion, we further refined the coding tree and looked for higher-level themes. Our analysis resulted in four themes: COMFORT, Control, Creativity and ImMersion. We present the contents of each theme below.

\section{Comfort}

Participants commented extensively on how the two versions of the system varied in terms of comfort of use. When prompted about the need to affix electrodes to their bodies to use the system, many users reported that they could not feel the electrodes while playing. One participant reflected that they forgot about the electrodes altogether:

\section{So, the electrodes did not disturb me at all. I didn't perceive it. (P10)}

Another aspect of comfort was the choice of the thumb as the finger used for activation and the possibility of using other fingers for modulation. The quote below illustrates how participants saw the use of other fingers as an opportunity for increased ease of use, which also may have decreased mental coordination:

Occasionally, I thought it would make sense from a musical perspective. However, at that point in time, I did not press the key with my thumb, so I forgot to use the thumb for activation. (P12)

Participants also reflected on the fact that playing the piano with pitch effects required additional mental coordination. This was true for both Electromyography and Pitch Wheel. One participant reported needing to think ahead about what notes to play:

I tried to include this [EMG], but it influenced me in a way that required me to rethink, which sometimes caused me to miss the next couple of notes that I wanted to play. Sometimes I lost notes cause I had to concentrate to keep up the movement. (P6)

Similarly, the pitch wheel also required extra time for reflecting on what to play. Using the wheel changed the perception of the instrument. Some users needed to play slower in order to have enough time to activate the effect. This is illustrated by the following remark:

Simply put, I had to pause playing to alter the tone myself. Also, that I altered the chords. It felt more like a string instrument than a keyboard instrument. (P11)

\section{Control}

Another important aspect for participants was the perceived control over the modulation. For most participants using the pitch wheel, modulation felt cumbersome and it was difficult to achieve a desirable vibrato effect:

On the other hand, it [pitch wheel] is also difficult. Especially that light vibrato is not that easy to achieve. Reproducing this, I experienced it as much more taxing than using the gesture [EMG]. (P8)

While using our system enabled players to stay in control, they also reported on the lack of fine-grained control when needed:

Using EMG, what I really like is that I could be focused on what I was doing, and because this [referring to the pitch wheel] will be very far from when I'm hitting the piano. I could focus on what I was playing. I could just do some kind of thing on the vibrato like you said, but I wasn't able to precisely control the modulation. (P2)

Being aware of the nature of the EMG-based modulation, participants formulated new ideas on how to control the interaction with the piano keyboard using muscle activation:

For example, when I wiggle my ears, I can trigger an arpeggio. (P9)

Relating to the previous theme COMFORT, the participants associated being able to use more fingers for activating the modulation with increased control: 
Yes, I think it would help me because there are a lot of tracks in which you may want to use modulation on a specific note, but on this note you're not supposed to use your thumb. Yes, I think it would be great to be able to do it with other fingers. (P2)

\section{Creativity}

One aspect on which we focused in this work was whether an EMG-based system could provide creative support to express oneself. This theme describes the participants' impressions of creativity during the study. Participants reported that our prototype encouraged them to explore different aspects of the sound modulation:

Well, yes because I wanted to try out on which notes the vibrato would soon sound the best. (P2)

You can use it for the sound [the note played] itself, but you can also use it to modify other notes while holding down a note. (P5)

Similarly, this led to a feeling of increased creativity due to the added expressive range after successfully integrating it into the play process:

I think it offered me more possibilities. I also could utilize it well. It was unfamiliar at first so I tried to find fitting passages where I thought it would sound cool, and that worked really well. I can imagine that this [EMG] can help me to convey what I want to express. (P5)

More experienced players commented on the lack of controllable parameters for the EMG-based version. For them, the greater expressive range of the pitch wheel offered more ideas to pursue, albeit being more difficult to achieve:

I had the feeling that especially if I do something like this [uses pitch wheel modulation]. Instead of climbing to $C, I$ can use the pitch wheel modulation. I cannot do that with the other gesture [EMG]. Because I had absolute control, I could develop new ideas, where it was clear that I could not do this with the other system [EMG]. Hence, it helped me to create more ideas. But they were very difficult to achieve, too. (P8)

We identified that the fun associated with using something novel was one of the main driving factors for increased creativity when using the EMG-based system:

One could create a kind of vibrato, like when using a cello. And I think that is something I have been missing for the piano. That's why I like it very much. (P12)

\section{Immersion}

The last theme resulting from our analysis is IMMERSION. Being immersed is one of the main drivers for getting into a flow state during play. Participants reported a limited and disturbed feeling of immersion when using the pitch wheel:

My left hand was always occupied with that thing [the pitch wheel]. I do not know of any pieces that would allow me to switch in between. (P6)
Using the EMG-based modulation supported many players in achieving a flow-like state and immersing themselves in their improvisation:

For sure. I had the feeling to get into a flow state very quickly, because the other method [pitch wheel] interrupted me motion-wise. That always pulled me out of the flow. I also had the feeling that I struggled to stay on tempo and that pulled me out. With the other system [EMG], I did not have this feeling. I experienced a more flow-like feeling. (P8)

Participants commented how flow could be achieved. Firstly, the EMG-based system allowed for an immediate and nondisruptive integration into the participants' play style:

It was simply very intuitive. If I wanted to use it, it just worked. (P11)

Secondly, due to the seamless integration, the EMG-based system enabled participants to play freely:

And I think, when I incorporated it into my play style, it enabled me to play freely. This method was much better than the other one [pitch wheel], because it was much more effort for me to reach. (P8)

\section{DISCUSSION}

Our investigation showed that there are multiple facets to consider when designing for EMG-based systems, especially as a secondary modality.

\section{Input Ambiguity Stimulates Playful Exploration}

In our first research question, we looked at technical feasibility and the system's impact on playing style and interaction range. Related work has already demonstrated the technical feasibility by recognizing fine-grained finger motions [25]. We have confirmed this observation in our study, partly answering RQ1, additionally supported by our classification results on a reduced set of electrode channels. The decrease in performance was marginal, making the required setup with only one or two channels feasible.

Our work still has technical challenges. However, the nature of the system is advantageous. We observed that participants learned to control the activation and adapted it to their play style. While this initially placed a cognitive burden on players, eventually finding the right movement to trigger activation was rarely a problem. Yet, this might indicate that players should be given even more freedom in defining their own activation gestures, adding to the second aspect of RQ1.

The inherent ambiguity of our system has proven beneficial for our scenario [16] as it allowed players to define their own interaction interface for the piano keyboard. This observation is also supported through the increased feeling of immersion that participants reported for the EMG-based system, indicating that such a system can support a more seamless integration. It provides increased interaction possibilities while being part of the task language. This provides valuable input for RQ2. Consequently, future designs that use EMG can enable playful explorations through the ambiguity that is inherent to using 
EMG as a second input modality, as illustrated in the CREATIVITY theme in our interview analysis.

\section{EMG Can Enable Seamless Integration}

For our second research question RQ2, we looked at design requirements that need to be fulfilled to achieve seamless integration and whether these can be applied to other existing motor tasks. To do so, we investigated aspects of piano play related to user experience and motives for playing. In our experiment, we identified hedonic goals such as enjoyment as one main driver to play the piano, while participants also rated their feeling of being immersed and achieving results worth the effort as important.

Thus, an auxiliary input system should strengthen these aspects without negatively affecting play performance. While our EMG-based system supported enjoyment and exploration during the improvisation task, it only slightly fostered musical creativity as indicated in our questionnaires. However, one important advantage compared to the pitch wheel modulation is the fact that the utilized gesture activation was rated much higher by players in terms of intuitiveness. For them, the connection between the movement and the resulting sound modulation was straightforward and immediate to apply, facilitating seamless integration. It remains to be investigated, whether long-term use can achieve the same level of integration.

\section{Increasing the Expressive Range Can Increase Perceived Expressiveness}

In this work, we made the design decision to provide only one sound modulation using the thumb limiting the increase expressive range to reduce cognitive demand. During our interviews, it became clear that a cognitive cost was still present. Participants reported the need for mental coordination to correctly use the EMG-based modulation. On the other hand, providing only a predefined effect was appreciated by most participants as the need to control more parameters overloaded most of them. This was evident when looking at the pitch wheel condition, which was often criticized for its high complexity. Advanced players appreciated the greater control over the sound modulation when using the pitch wheel.

We believe that extending the system in future work to allow modulation with all fingers is cognitively feasible and may even be beneficial in reducing the temporal demand for coordination (RQ2). Especially as skills improve, players might appreciate greater control and flexibility (analogous to using a pitch wheel), but can still profit from the seamless integration through EMG. This long-term use should contrast methods used in advanced keyboard setups such as sticks or pedals as baseline as well.

\section{Design Requirements for EMG-Based Interaction}

Ultimately, using our EMG-based system fulfills the criteria that we outlined in our design section. It enables players to stay immersed and continue a flow-like state during usage $[11,33]$, while providing increased perceived expressiveness. Further, the increased expressive range is realized without added complexity through seamless integration allow- ing for good control using already established "task language". Our observations in this experiment provide an extensive answer for RQ2. Thus, design for EMG-based interaction should balance the amount of control offered to the user.

\section{CONCLUSION}

In this paper, we presented a way to increase the expressive range of a piano keyboard that does not rely on modifying the instrument. Leveraging Electromyography, we enable players to augment their playing style through motion activated sound modulation. Post-hoc interviews and questionnaires confirmed the capabilities of EMG-based activation to support the players' musical flow and creativity, while not hindering play in any way.

This work aimed at validating whether EMG can support seamless integration into existing systems to extend the range of interaction. Hence, we focused on one specific modulation in conjunction with an associated gesture motion. As reported by the participants, extending the system to include multiple fingers governing multiple effects should be addressed by further research. Even more so, extending our approach to other body parts could create a form of embodied interaction, venturing into the realms of biophysical music [13].

Our successful integration of EMG as a secondary modality into an already well-established motor task showed that EMG does have the potential to enable additional interactions with tangible artifacts without altering the existing artifact or introducing new ones. We envision that these findings will inspire further research leveraging the advantages of integrating EMG into motor tasks.

\section{ACKNOWLEDGMENTS}

This research is financially supported by the European Union's Horizon 2020 Programme under ERCEA grant no. 683008 AMPLIFY. Part of this research was supported by the German Research Foundation (DFG) within the project $\mathrm{C} 02$ of SFB/Transregio 161. 


\section{REFERENCES}

[1] E. D. Adrian and D. W. Bronk. 1929. The Discharge of Impulses in Motor Nerve Fibres. J Physiol 67, 2 (March 1929), i3-151.

[2] D. T. Barry, K. E. Gordon, and G. G. Hinton. 1990. Acoustic and Surface EMG Diagnosis of Pediatric Muscle Disease. Muscle Nerve 13, 4 (April 1990), 286-290. DOI :

http://dx.doi.org/10.1002/mus. 880130403

[3] Ronald A. Beghetto. 2006. Creative Self-Efficacy: Correlates in Middle and Secondary Students. Creativity Research Journal 18, 4 (2006), 447-457. DOI : http://dx.doi .org/10.1207/s15326934crj1804_4

[4] Steve Benford, Holger Schnädelbach, Boriana Koleva, Rob Anastasi, Chris Greenhalgh, Tom Rodden, Tom Rodden, Jonathan Green, Ahmed Ghali, Tony Pridmore, Bill Gaver, Andy Boucher, Brendan Walker, Sarah Pennington, Albrecht Schmidt, Hans Gellersen, Anthony Steed, and Anthony Steed. 2005. Expected, Sensed, and Desired: A Framework for Designing Sensing-based Interaction. ACM Trans. Comput.-Hum. Interact. 12, 1 (March 2005), 3-30. DOI :

http://dx.doi.org/10.1145/1057237.1057239

[5] Ann Blandford, Dominic Furniss, and Stephann Makri. 2016. Qualitative HCI research: Going behind the scenes. Synthesis lectures on human-centered informatics 9, 1 (2016), 1-115.

[6] Joseph D. Bronzino. 2006. The Biomedical Engineering Handbook, Third Edition. Taylor \& Francis.

[7] Baptiste Caramiaux, Marco Donnarumma, and Atau Tanaka. 2015. Understanding Gesture Expressivity Through Muscle Sensing. ACM Trans. Comput.-Hum. Interact. 21, 6 (Jan. 2015), 31:1-31:26. DOI : http://dx.doi.org/10.1145/2687922

[8] Erin Cherry and Celine Latulipe. 2014. Quantifying the Creativity Support of Digital Tools Through the Creativity Support Index. ACM Trans. Comput.-Hum. Interact. 21, 4 (June 2014), 21:1-21:25. DOI : http://dx.doi.org/10.1145/2617588

[9] Enrico Costanza, Samuel A. Inverso, and Rebecca Allen. 2005. Toward Subtle Intimate Interfaces for Mobile Devices Using an EMG Controller. In Proceedings of the SIGCHI Conference on Human Factors in Computing Systems (CHI '05). ACM, New York, NY, USA, 481-489. DOI :

http://dx.doi.org/10.1145/1054972.1055039

[10] Enrico Costanza, Samuel A. Inverso, Rebecca Allen, and Pattie Maes. 2007. Intimate Interfaces in Action: Assessing the Usability and Subtlety of Emg-Based Motionless Gestures. In Proceedings of the SIGCHI Conference on Human Factors in Computing Systems (CHI '07). ACM, New York, NY, USA, 819-828. DOI : http://dx.doi.org/10.1145/1240624.1240747

[11] Mihaly Csikszentmihalyi. 1991. Flow: The Psychology of Optimal Experience. Harper Perennial, New York, NY.
[12] Örjan de Manzano, Töres Theorell, László Harmat, and Fredrik Ullén. 2010. The psychophysiology of flow during piano playing. Emotion 10, 3 (2010), 301-311. DOI : http://dx.doi.org/10.1037/a0018432

[13] Marco Donnarumma. 2011a. XTH SENSE. (2011). https://marcodonnarumma.com/works/xth-sense/

[14] Marco Donnarumma. 2011b. XTH SENSE: a study of muscle sounds for an experimental paradigm of musical performance. In $I C M C$.

[15] Marco Donnarumma, Baptiste Caramiaux, and Atau Tanaka. 2013. Muscular Interactions Combining EMG and MMG sensing for musical practice. In Proceedings of the Conference on New Interfaces for Musical Expression (NIME'13).

[16] William W. Gaver, Jacob Beaver, and Steve Benford. 2003. Ambiguity As a Resource for Design. In Proceedings of the SIGCHI Conference on Human Factors in Computing Systems (CHI '03). ACM, New York, NY, USA, 233-240. DOI : http://dx.doi.org/10.1145/642611.642653

[17] Aristotelis Hadjakos and Simon Waloschek. 2014. ViP: Controlling the Sound of a Piano with Wrist-Worn Inertial Sensors. (2014), 2.

[18] Sandra G. Hart. 2006. Nasa-Task Load Index (NASA-TLX); 20 Years Later. Proceedings of the Human Factors and Ergonomics Society Annual Meeting 50, 9 (2006), 904-908. D0I : http://dx.doi .org/10.1177/154193120605000909

[19] Sandra G. Hart and Lowell E. Staveland. 1988. Development of NASA-TLX (Task Load Index): Results of empirical and theoretical research. In Human mental workload. North-Holland, Oxford, England, 139-183. DOI : http://dx.doi .org/10.1016/S0166-4115(88)62386-9

[20] G. Hefftner and G. G. Jaros. 1988. The Electromyogram (EMG) as a Control Signal for Functional Neuromuscular Stimulation. II. Practical Demonstration of the EMG Signature Discrimination System. IEEE Transactions on Biomedical Engineering 35, 4 (April 1988), 238-242. DOI : http://dx.doi .org/10.1109/10.1371

[21] G. Hefftner, W. Zucchini, and G. G. Jaros. 1988. The Electromyogram (EMG) as a Control Signal for Functional Neuromuscular Stimulation. I. Autoregressive Modeling as a Means of EMG Signature Discrimination. IEEE Transactions on Biomedical Engineering 35, 4 (April 1988), 230-237. DOI : http://dx.doi.org/10.1109/10.1370

[22] A. Hernandez Arieta, R. Katoh, H. Yokoi, and Y. Wenwei. 2006. Development of a Multi-DOF Electromyography Prosthetic System Using the Adaptive Joint Mechanism. (2006). DOI : http://dx.doi.org/10.1533/abbi.2005.0060 
[23] Veronika Huta and Richard M. Ryan. 2010. Pursuing Pleasure or Virtue: The Differential and Overlapping Well-Being Benefits of Hedonic and Eudaimonic Motives. J Happiness Stud 11, 6 (Dec. 2010), 735-762. DOI : http://dx.doi . org/10.1007/s10902-009-9171-4

[24] Wonil Hwang and Gavriel Salvendy. 2010. Number of People Required for Usability Evaluation: The $10 \pm 2$ Rule. Commun. ACM 53, 5 (May 2010), 130. DOI : http://dx.doi.org/10.1145/1735223.1735255

[25] Jakob Karolus, Hendrik Schuff, Thomas Kosch, PawełW. Wozniak, and Albrecht Schmidt. 2018. EMGuitar: Assisting Guitar Playing with Electromyography. In Proceedings of the 2018 Designing Interactive Systems Conference (DIS '18). ACM, New York, NY, USA, 651-655. DOI :

http://dx.doi.org/10.1145/3196709.3196803

[26] R. Benjamin Knapp and Hugh S. Lusted. 1990. A Bioelectric Controller for Computer Music Applications. Computer Music Journal 14, 1 (1990), 42-47. DOI : http://dx.doi.org/10.2307/3680115

[27] C. J. De Luca. 1979. Physiology and Mathematics of Myoelectric Signals. IEEE Transactions on Biomedical Engineering BME-26, 6 (June 1979), 313-325. DOI : http://dx.doi.org/10.1109/TBME. 1979. 326534

[28] Andrew P. McPherson, Adrian Gierakowski, and Adam M. Stark. 2013. The Space Between the Notes: Adding Expressive Pitch Control to the Piano Keyboard. In Proceedings of the SIGCHI Conference on Human Factors in Computing Systems (CHI'13). ACM, New York, NY, USA, 2195-2204. DOI : http://dx.doi.org/10.1145/2470654.2481302

[29] Elisa D. Mekler and Kasper Hornbæk. 2016. Momentary Pleasure or Lasting Meaning?: Distinguishing Eudaimonic and Hedonic User Experiences. In Proceedings of the 2016 CHI Conference on Human Factors in Computing Systems (CHI'16). ACM, New York, NY, USA, 4509-4520. DOI :

http://dx.doi.org/10.1145/2858036.2858225

[30] Roberto Merletti and Dario Farina. 2016. Surface Electromyography: Physiology, Engineering and Applications. John Wiley \& Sons.

[31] Roberto Merletti and Philip A. Parker. 2004. Electromyography: Physiology, Engineering, and Non-Invasive Applications. John Wiley \& Sons.

[32] Sarah Nicolls. 2010. Seeking Out the Spaces Between: Using Improvisation in Collaborative Composition with Interactive Technology. Leonardo Music Journal 20 (Dec. 2010), 47-55. DOI : http://dx.doi.org/10.1162/LMJ_a_00012

[33] R. Parncutt and G. McPherson. 2002. The Science and Psychology of Music Performance: Creative Strategies for Teaching and Learning. Oxford University Press.
[34] D. Peleg, E. Braiman, E. Yom-Tov, and G. F. Inbar. 2002. Classification of Finger Activation for Use in a Robotic Prosthesis Arm. IEEE Transactions on Neural Systems and Rehabilitation Engineering 10, 4 (Dec. 2002), 290-293. DOI :

http://dx. doi.org/10.1109/TNSRE. 2002.806831

[35] T Scott Saponas, Desney S. Tan, Dan Morris, and Ravin Balakrishnan. 2008. Demonstrating the Feasibility of Using Forearm Electromyography for Muscle-Computer Interfaces. In Proceedings of the SIGCHI Conference on Human Factors in Computing Systems (CHI '08). ACM, New York, NY, USA, 515-524. DOI : http://dx.doi.org/10.1145/1357054.1357138

[36] T. Scott Saponas, Desney S. Tan, Dan Morris, Ravin Balakrishnan, Jim Turner, and James A. Landay. 2009. Enabling Always-Available Input with Muscle-Computer Interfaces. In Proceedings of the 22Nd Annual ACM Symposium on User Interface Software and Technology (UIST '09). ACM, New York, NY, USA, 167-176. DOI : http://dx.doi.org/10.1145/1622176.1622208

[37] Marian Theiss, Philipp M. Scholl, and Kristof Van Laerhoven. 2016. Predicting Grasps with a Wearable Inertial and EMG Sensing Unit for Low-Power Detection of In-Hand Objects. In Proceedings of the 7th Augmented Human International Conference 2016 (AH '16). Association for Computing Machinery, New York, NY, USA, Article Article 4, 8 pages. DOI: http://dx.doi.org/10.1145/2875194.2875207

[38] Pamela Tierney and Steven M. Farmer. 2002. Creative Self-Efficacy: Its Potential Antecedents and Relationship to Creative Performance. The Academy of Management Journal 45, 6 (2002), 1137-1148.

[39] Alejandro Van, Baptiste Caramiaux, and Atau Tanaka. 2014. PiaF: A Tool for Augmented Piano Performance Using Gesture Variation Following. (2014), 4.

[40] Scott R. Vrana. 1993. The Psychophysiology of Disgust: Differentiating Negative Emotional Contexts with Facial EMG. Psychophysiology 30, 3 (May 1993), 279-286. DOI :

http://dx.doi.org/10.1111/j.1469-8986.1993.tb03354.x

[41] Katrin Wolf and Jonas Willaredt. 2015. PickRing: Seamless Interaction through Pick-up Detection. In Proceedings of the 6th Augmented Human International Conference (AH'15). Association for Computing Machinery, New York, NY, USA, 13-20. DOI : http://dx. doi .org/10.1145/2735711.2735792

[42] Qi Yang and Georg Essl. 2012. Augmented Piano Performance using a Depth Camera. (2012), 2. 